Collection SFN 8 (2007) 43-60

(C) EDP Sciences, Les Ulis

DOI: $10.1051 / \mathrm{sfn}: 2007005$

\title{
Étude des propriétés dynamiques des fluides confinés par diffusion quasiélastique de neutrons
}

C. Alba-Simionesco

\author{
LCP, Bâtiment 349, Université de Paris 11, 91405 Orsay Cedex, France
}

\begin{abstract}
Résumé. La spectroscopie par diffusion quasi-élastique de neutrons offre la possibilité d'étudier les propriétés dynamiques de liquides confinés à l'échelle de quelques nanométres dans des matériaux poreux plus ou moins rigides. Le principe de ces mesures et les différentes techniques associées sont rapidement exposés ainsi que les fonctions de diffusion. L'état actuel des connaissances ne fait pas apparaître d'interprétation consensuelle et différents modéles sont à considérer ; il est essentiel de se situer selon deux cas limites : -où la dynamique n'est pas intrinséquement modifiée, mais l'étendue des déplacements est limitée, ou bienoù la dynamique est profondément modifiée par des effets de taille finie, des effets de surface et demande une nouvelle paramétrisation. Le cas particulier de fluides moléculaires dans des mésopores silicatés est présenté comme illustration.
\end{abstract}

\section{CONTEXTE DE L'ÉTUDE : EFFET DU CONFINEMENT NANOSCOPIQUE SUR LESS PROPRIÉTÉS DES FLUIDES MOLÉCULAIRES}

De nombreux thémes de recherche fondamentale actuels et d'applications industrielles mettent en situation un élément confiné dans un autre à l'échelle de quelques nanométres et les nanosciences connaissent de nos jours un développement considérable. Des domaines tels que la catalyse hétérogène, la filtration, la chromatographie, le relargage contrôlé de principe actif, l'exploitation pétrolière et gazière, le stockage à long terme des déchets nucléaires mais aussi les projets de stockage de combustibles, la diffusion contrôlée des médicaments dans l'industrie pharmaceutique, etc... nécessitent de comprendre les mécanismes mis en jeu tant du point du vue de la stabilité hydrothermale et mécanique des matériaux mésoporeux utilisés, que du point de vue des propriétés de l'élément confiné lui-même. L'intérêt fondamental de ce domaine réside dans le désir de comprendre et de desceller de nouveaux phénomènes liés aux effets de taille finie et de dimensionalité réduite ainsiqu'aux forces at aux interactions avec une surface.

Les exemples sont nombreux et parmi eux les fluides confinés dans des nanopores, des micelles ou bien entre deux plans dans les machines à force de surface, ou bien encore à l'interface de macromolécules d'intérêt biologique. Les matériaux micro et méso poreux (le terme nano n'est pas attribué pas l'IUPAC), synthétisés récemment avec des largeurs proches de $2 \mathrm{~nm}$ et de 2-50 nm respectivement, peuvent avoir des géométries trés différentes : planes (e.g. fibres de carbone activé, «boron nitride » micropore, argiles), cylindriques (e.g. MCM-41 en 1992, SBA-15 en 1998) ou bien sphériques (e.g. aérogels, mousses), $c f$ table 1. Réduire l'espace de confinement jusqu'aux dimensions des forces intermoléculaires conduit à des variations significatives des températures de transition de phases condensées telles que la fusion ou la cristallisation, et dans certains cas met en évidence de nouvelles phases, amorphes, induites par la surface et le confinement. La phase confinée est nommée adsorbât et le matériau hôte adsorbant. Comme pour les mécanismes de mouillage et de condensation capillaire, on peut s'attendre à trouver pour les phases condensées une compétition forte entre les forces d'interaction adsorbât-adsorbât et adsorbât-adsorbant (ou dans notre cas ici fluide-fluide et fluide-mur). En effet quand un système (molécule ou fluide) est confiné dans des pores de petites tailles ses propriétés thermodynamiques, structurales et dynamiques sont substantiellement modifiées. Ainsi les questions se ramènent à comprendre et modéliser comment l'existence d'un confinement spatial, tant dans sa morphologie, sa topologie, que dans ses propriétés physico-chimiques, influence l'évolution des propriétés 
thermodynamiques, dynamiques et structurales de liquides moléculaires confinés et détermine le statut de transitions de phases (gaz-liquide, liquide-liquide, liquide-solide), avec en corollaire les questions inhérentes à l'origine et la nature des états métastables, notamment l'émergence de transitions vitreuses.

Les travaux engagés tant sur le plan expérimental que théorique se heurtent à des difficultés majeures ; la nature de ces dernières est souvent différente, ce qui encourage un travail selon les deux aspects et rend leurs apports complémentaires. Du point de vue expérimental, une des difficultés provient de l'imprécision de critéres pour la détermination -de la structure des phases condensées (cristal ou amorphe), -de l'état thermodynamique stable ou métastable (verre ou liquide visqueux), -ou simplement pour prendre en compte proprement les caractéristiques et les défauts des matériaux hôtes, les mesures se ramenant à une valeur moyenne de tout ce qu'il y a et se passe à l'intérieur du pore. Les phénomènes sont en général très étalés et moins bien résolus qu'en volume et le type de transition de phase difficile à attribuer ; le niveau des compétitions entre des effets de surface et de taille finie, entre désordre gelé ou auto-induit, dépend du phénomène considéré et nécessitent toujours des études par plusieurs sondes.

En ce qui concerne la compréhension du phénomène de vitrification, la recherche d'une longueur mésoscopique, associée au processus de ralentissement visqueux et aux hétérogénéités dynamiques observées dans le liquide, a fait l'objet de nombreuses études en confinement. L'état actuel des connaissances ne fait pas apparaître d'interprétation consensuelle dans ce domaine et en particulier l'effet de taille finie, la morphologie des matériaux de confinement, les interactions du fluide avec la surface, la structure et l'état thermodynamique du fluide confiné sont autant de paramètres dont le rôle est mal défini. En confinant un système, nous introduisons une longueur supplémentaire par le biais d'un paramètre extérieur, un diamètre poreux que l'on peut faire varier.

La présence d'un mur rigide induit un ralentissement graduel des molécules relatif à leur distance au mur ; de ce ralentissement dans l'espace on peut extraire une longueur de nature coopérative $\xi_{\text {coop }}$, qui serait intrinsèque à chaque système et comparable, en espèce, à celle du liquide en volume, dès lors que notre système est à la bonne densité, ou ne présente aucune modification notable de ses propriétés structurales induites par confinement. On peut alors tout naturellement se poser la question de sa mise en évidence qualitative voire quantitative en confinant un liquide. Malheureusement, nous avons constaté qu'il était nécessaire d'obéir à un certain nombre de conditions pour qu'une telle situation soit rencontrée, et que ces conditions ne peuvent pas être satisfaites. Les interactions fluide-mur sont elles aussi modifiable par traitement chimique (cf Figure 1) ; elles entraînent la formation d'une couche de molécules à la surface, stabilisée plus ou moins bien selon le degré d'attractivité ou de répulsion, mais qui représentera au minimum une monocouche. La taille de la molécule, $\sigma$, apparaît alors comme un paramètre supplémentaire important à prendre en compte quand on veut comparer les systèmes. La présence d'une interface ralentie a pour conséquence un effet de volume exclu à prendre en compte dans les propriétés structurales et un ralentissement des molécules prés du mur, provoquant de fait une inhomogénéité spatiale des propriétés dynamiques.
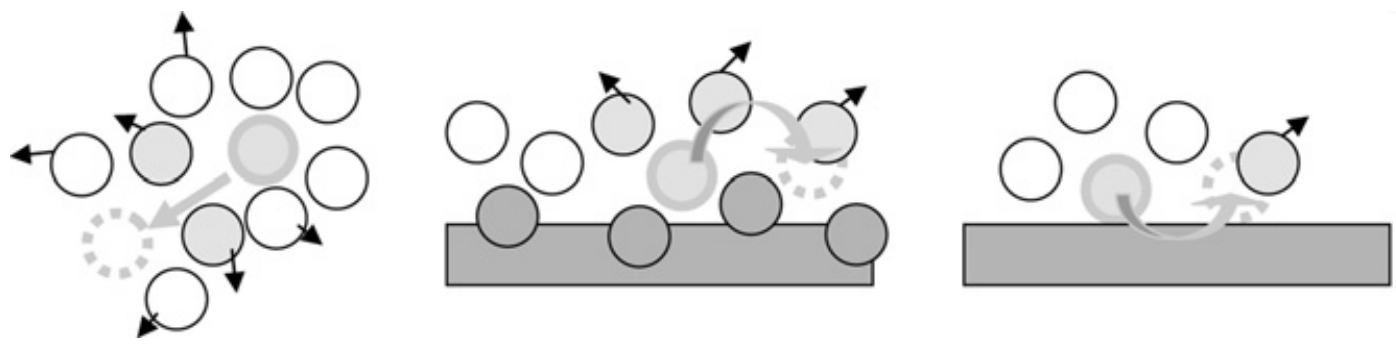

Figure 1. (De gauche à droite) processus de diffusion au sein d'un liquide en volume, près d'un mur rugueux ralentissant fortement les molécules et près d'un mur lisse pouvant faciliter la diffusion. 
Avant toute analyse, il est indispensable de séparer les différentes contributions provenant du matériau en caractérisant celui-ci au mieux (distribution de tailles et de formes de pores, la nature de l'interaction mur-liquide). Le confinement peut faire apparaître des propriétés singulières du liquide, qu'il est important de pouvoir comprendre, et de relier à des paramètres caractéristiques du milieu confinant. Il faut donc connaître au mieux ces matériaux (voire jusqu'à leur synthèse) et engager des études et faisant varier conjointement, la température et la taille de pore comme paramètres pilotes, la nature des interactions de surface et leur topologie, mais aussi imposer une longueur suppose connaître aussi le système en volume autour de cette taille. Cela rend la construction d'un modèle ardue et il est essentiel de se situer selon deux cas limites : - où la dynamique n'est pas intrinsèquement modifiée, mais l'étendue des déplacements est limitée, ce qui conduit à tronquer l'espace et introduire seulement des effets de volume exclu, la contribution du matériau pouvant être aisément soustraite, ou bien -où la dynamique est profondément modifiée par des effets de taille finie, des effets de surface et demande une nouvelle paramétrisation.

Table 1. Divers matériaux nanoscopiques rigides.

\begin{tabular}{|l|l|l|l|}
\hline Porous material & Chimie de Surface & Forme du Pore & Taille du Pore /nm \\
\hline Ordered, Regular: & & & \\
\hline Zeolite Aluminosilicate & $\mathrm{O}, \mathrm{Si}, \mathrm{Al}$ & Cylinders, Cages & $0.3-1$ \\
\hline Zeolite Aluminophosphate & $\mathrm{O}, \mathrm{P}, \mathrm{Al}$ & Cylinders & $0.8-1.3$ \\
\hline Carbon nanotube & $\mathrm{C}$ & Cylinders & $0.5-10$ \\
\hline MCM-41 & $\mathrm{Si}, \mathrm{O}(\mathrm{H})$ & Cylinders & $1-5$ \\
\hline SBA-15 & $\mathrm{Si}, \mathrm{O}(\mathrm{H})$ & Cylinders (connected) & $3-10$ \\
\hline Porous silicon & $\mathrm{SiH}_{x}(x=1,2,3)$ & Polygonal cylinders & $5-50$ \\
\hline & & & \\
\hline Disordered: & & & \\
\hline Porous glass & $\mathrm{Si}, \mathrm{O}(\mathrm{H})$ & Distorted cylinders & $2.5-10^{4}$ \\
\hline Silica Xerogel, oxides & $\mathrm{Si}, \mathrm{O}(\mathrm{H}), \ldots$ & Distorted cylinders & $1-100$ \\
\hline Silica aerogel & $\mathrm{Si}, \mathrm{O}(\mathrm{H})$ & Pore voids & $5-50$ \\
\hline Carbon aerogel & $\mathrm{C}$ & Slits and pore voids & $1.5-60$ \\
\hline Activated Carbon Fibers & $\mathrm{C}$ & Slits & $0.6-1.3$ \\
\hline Pillared clay & $\mathrm{O}, \mathrm{Si}, \mathrm{Al}, \ldots$ & Slits and pillars & $0.5-2$ \\
\hline
\end{tabular}

\section{PROPRIÉTÉS DYNAMIQUES PAR DIFFUSION QUASI-ÉLASTIQUE DE NEUTRONS}

\subsection{Que mesure-t-on pendant une expérience de diffusion de neutrons ?}

Les neutrons sont produits dans des réactions nucléaires pouvant être le choc de particules chargées (électrons, protons) accélérées sur une cible, la fusion ou la fission. Le choix de la technique utilisée prend en compte le coût de production des neutrons, c'est-à-dire le rapport prix en MeV par neutron (ce prix peut comprendre par exemple la puissance nécessaire pour accélérer des particules, le prix de refroidissement d'un réacteur nucléaire, etc...). En pratique les réactions les plus rentables sont la fission et la spallation. Les neutrons sont produits dans des réactions nucléaires pouvant être le choc de particules chargées (électrons, protons) accélérées sur une cible, la fusion ou la fission. Dans la réaction de fission, un neutron thermique est absorbé par un noyau ${ }^{235} U$. Le noyau passe alors dans un état très excité, et se divise en quelques fragments de fission (atomes semi-lourds) et 2 à 5 neutrons rapides. En moyenne, chaque réaction donne 2.5 neutrons, dont 1.5 sont nécessaires pour alimenter la réaction 
en chaîne : chaque réaction donne donc un neutron utilisable, d'énergie cinétique de l'ordre de $2 \mathrm{MeV}$. Ce système est celui utilisé au Laboratoire Léon Brillouin (CEA Saclay) (réacteur d'environ 14 MW), ainsi qu'à l'Institut Laue-Langevin (Grenoble) (réacteur d'environ 54 MW, 1MW correspond environ à une source de $3.10^{16}$ neutrons par seconde).

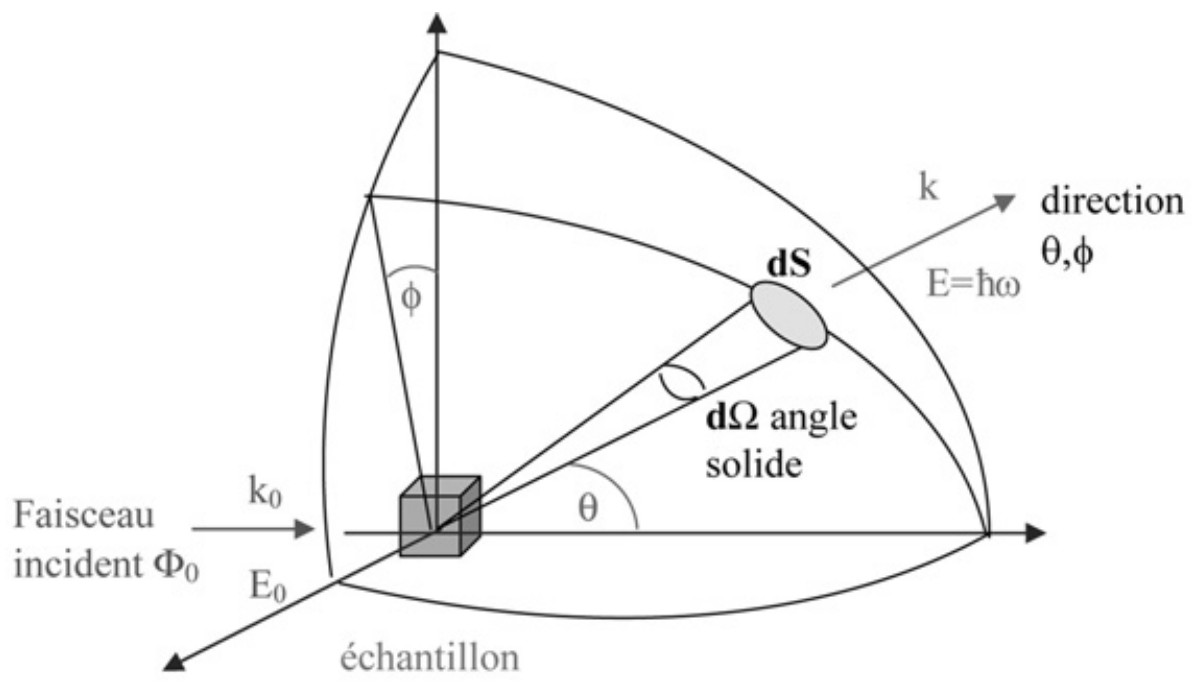

Figure 2. Schéma de principe d'une mesure par diffusion de neutrons.

Lorsque l'on réalise une expérience de diffusion de neutrons, on mesure la quantité de neutrons diffusés par un échantillon par seconde, $\sigma$, rapportée au flux incident $\Phi_{0}$ de neutrons par unité de surface $\left(\mathrm{cm}^{2}\right)$ et par seconde. Cette quantité totale n'est pas exploitable directement mais est différenciée pour un angle solide donné $d \Omega$ et une énergie diffusée comprise entre $E^{\prime}$ et $E^{\prime}+d E^{\prime}$. C'est la section efficace différentielle partielle qui s'exprime en barns par stéradian et par unité d'énergie :

$$
\frac{d^{2} \sigma}{d \Omega d E^{\prime}}=\frac{1}{\Phi_{0}} \frac{\text { nombre de neutrons diffusé s par seconde dans } d \Omega \text { et } d E^{\prime}}{d \Omega d E^{\prime}}
$$

Par convention $E=\hbar \omega$ est positif lorsque le neutron cède de l'énergie au système.

\section{Interaction noyau-neutron}

Tout comme la lumière, les neutrons présentent la caractéristique de dualité onde-corpuscule. Le mouvement d'un neutron se déduit des lois de propagation de l'onde associée. Nous nous appuierons donc sur cette dualité pour présenter la diffusion de neutrons. Non chargé, le neutron, de par son spin, interagit de manière magnétique. Dans les cas de fluides confinés considérés ici, les systèmes étudiés ne possèdent pas d'électrons célibataires susceptibles de créer un moment magnétique. La seule interaction magnétique qui a lieu est donc l'interaction du spin du neutron avec ceux des noyaux atomiques.

L'interaction noyau- neutron est une force à très courte portée (de l'ordre de $10^{-13} \mathrm{~cm}$ ), que l'on représente par le pseudo-potentiel de Fermi $V(r)$ avec ..la position du neutron, ..la position de l'atome, et $b$ la longueur de diffusion de l'atome. La force de l'interaction, représentée par la longueur de diffusion, dépend donc de la force de l'interaction spin / spin. Tout d'abord, les atomes ne portent pas tous le même spin. On a ainsi, par exemple, un spin de 1/2 pour l'Hydrogène, 1 pour le Deutérium, et 0 pour le ${ }^{12}$.. L'interaction dépend donc de l'espèce chimique, et de l'isotope considéré. De plus, un atome peut se trouver dans différents états de spins : H peut ainsi être dans l'état $+1 / 2$ ou $-1 / 2$. 
Les interactions résultantes seront différentes. La force de l'interaction neutron/noyau dépend donc de l'espéce chimique, de l'isotope considéré, et de l'état de spin.

Dans le cas où tous les atomes sont identiques, et dans le même état de spin, alors, les ondes diffusées par les atomes présentent entre elles une relation de phase définie. Les atomes font donc figure de sources secondaires cohérentes. La longueur de diffusion de chaque atome est alors dite cohérente, et on la notera $b c o h$. Entre les ondes diffusées de manière cohérente, il est possible d'avoir des interférences, c'est-à-dire d'additionner les amplitudes des ondes. La longueur d'onde des neutrons étant comparable aux distances intera-tomiques, ces figures d'interférence donnent des informations conséquentes sur la structure locale des systèmes. Dans le cas, maintenant, où tous les atomes ne sont pas identiques, ou pas tous dans le même état de spin, ils possèdent alors des longueurs de diffusion différentes. La relation de phase entre les ondes diffusées est alors variable, et ces atomes font figure de sources secondaires incohérentes. Les interférences ne peuvent alors avoir lieu, et l'on sommera uniquement les intensités des ondes. On schématise alors cela avec deux longueurs de diffusion, une cohérente bcoh et une incohérente binc. La longueur de diffusion cohérente d'un atome correspond à la moyenne des longueurs de diffusion des différents états de spin. Quant à la longueur de diffusion incohérente, elle rend compte des écarts à cette valeur moyenne. Les échantillons sont deutériés ou hydrogénés, selon que 1' on souhaite favoriser la diffusion cohérente ou incohérente.

Pour chaque élément, on définit

-sa section efficace totale :

$$
\sigma=4 \pi \overline{(b)^{2}}
$$

-sa section efficace cohérente

$$
\sigma_{c}=4 \pi(\bar{b})^{2}
$$

-sa section efficace incohérente

$$
\sigma_{i}=4 \pi \overline{(b)^{2}}-4 \pi(\bar{b})^{2}
$$

mais aussi sa section efficace d'absorption, dépendante de la longueur d'onde utilisée $\sigma_{\alpha}(\lambda)$. S'il s'agit maintenant d'une molécule de type $A_{x} B_{y}$, alors la section efficace de diffusion s'exprime comme suit : $\sigma_{A x B y}=x^{*} \sigma_{A}+y^{*} \sigma_{B}$

Dans le cas particulier de matériau confiné, l'expression de la section efficace de diffusion d'une « molécule moyenne » du système confiné (soit pour $\mathrm{N}$ molécules liquide $+\mathrm{N}$ molécules de matrice de type $\mathrm{SiO} 2$ par exemple) soit la pseudo-molécule réellement «vue » par les neutrons pour $1 \mathrm{~cm}^{3}$, se ramène à

$$
\sigma_{\text {conf }}=\sigma_{\text {liq }}+\left(N_{\text {mat }} / N_{\text {liq }}\right)^{*} \sigma_{\text {mat }}
$$

Une estimation des contributions respectives cohérente incohérente du matériau et du liquide est fournie dans la Table 2.

Table 2. Calcul des sections efficaces d'un système adsorbât (liquide o-terphenyl)/adsorbant (mésopores silicatés).

\begin{tabular}{|l|l|l|l|l|}
\hline systèmes & $\begin{array}{l}\sigma_{\text {coh }}(\text { matrice }) \\
/ 10^{22} \text { barn }\end{array}$ & $\begin{array}{l}\sigma_{\text {incoh }}(\text { matrice }) \\
/ 10^{22} \text { barn }\end{array}$ & $\begin{array}{l}\sigma_{\text {coh }}(\text { liq oTP }) \\
/ 10^{22} \text { barn }\end{array}$ & $\begin{array}{l}\sigma_{\text {incoh }}(\text { oTP }) \\
/ 10^{22} \text { barn }\end{array}$ \\
\hline $\mathrm{C}_{18} \mathrm{D}_{14}$ dans SBA-OD & 4.75 & 0.018 & 19.3 & 3.10 \\
\hline $\mathrm{C}_{18} \mathrm{H}_{14}$ dans SBA-OD & 4.96 & 0.018 & 12.7 & 120.5 \\
\hline $\mathrm{C}_{18} \mathrm{H}_{14}$ dans SBA-Si $\left(\mathrm{CH}_{3}\right)_{3}$ & 6.43 & 23.8 & 10.8 & 103.0 \\
\hline
\end{tabular}


Mis à part $\mathrm{le}^{12} C$, qui possède une longueur de diffusion incohérente nulle, du fait de son spin nul, les atomes étudiés possèdent souvent des longueurs de diffusion cohérente et incohérente non nulles. Les neutrons diffusés proviennent donc de la somme de ces contributions. Bien qu'il soit possible de mettre en valeur l'une ou l'autre par substitution isotopique, il n'est en général pas possible de les séparer complètement (cas des techniques de temps de vol, rétrodiffusion ou de trois axe). Cependant il existe d'autres techniques (temps de vol à polarisations de neutrons ou technique à écho de spin) où la polarisation préliminaire du faisceau de neutrons, puis l'analyse de la polarisation des neutrons diffusés, permet de séparer partiellement ces deux quantités. La combinaison de ces diverses techniques est donc souvent nécessaire quand le poids de ces sont équivalents.

La probabilité qu'un neutron $\left(E_{0}, k_{0}\right)$ soit diffusé avec $\left(E_{0}+\hbar \omega, k\right)$ revient à considérer les deux contributions, cohérente et incohérente :

$$
\left(\frac{d^{2} \sigma}{d \Omega d \omega}\right)=\left(\frac{d^{2} \sigma}{d \Omega d \omega}\right)_{\mathrm{coh}}+\left(\frac{d^{2} \sigma}{d \Omega d \omega}\right)_{\mathrm{inc}}
$$

avec respectivement pour $j \neq j$

$$
\begin{aligned}
& \left(\frac{d^{2} \sigma}{d \Omega d \omega}\right)_{\mathrm{coh}}=\frac{1}{2 \pi \hbar} \frac{k}{k_{0}} \frac{\sigma_{c}}{4 \pi} \sum_{j j^{\prime}} \int_{-\infty}^{+\infty}\left\langle e^{-i \vec{Q} \vec{R}_{j}(0)} e^{i \vec{Q} \vec{R}_{j^{\prime}}(t)}\right\rangle e^{-i \omega t} d t \\
& \left(\frac{d^{2} \sigma}{d \Omega d \omega}\right)_{\mathrm{inc}}=\frac{1}{2 \pi \hbar} \frac{k}{k_{0}} \frac{\sigma_{i}}{4 \pi} \sum_{j} \int_{-\infty}^{+\infty}\left\langle e^{-i \vec{Q} \vec{R}_{j}(0)} e^{i \vec{Q} \vec{R}_{j}(t)}\right\rangle e^{-i \omega t} d t
\end{aligned}
$$

\section{Diffusion quasi-élastique et inélastique des neutrons}

En plus des aspects de cohérence/incohérence de ce type d'expérience, les notions énergétiques sont à prendre en compte. En effet, ou bien les neutrons diffusés ont la même énergie que les neutrons incidents (diffusion élastique), ou bien ils ont subi un gain ou une perte d'énergie (diffusion quasiélastique et inélastique). C'est ainsi que Shull et Brockhouse, prix Nobel de physique 1994, ont défini la diffusion neutronique : 'neutrons tell you where the atoms are and what the atoms do'. Les neutrons possédent donc une longueur d'onde comparable aux distances inter-atomiques et une énergie comparable aux

\begin{tabular}{|c|c|c|}
\hline Quasi-élastique & Inélastique & $\begin{array}{l}\mu \mathrm{eV}-\mathrm{meV} \\
10^{-9} 10^{-12} \text { seconde }\end{array}$ \\
\hline $\begin{array}{l}\text { Mouvements de } \\
\text { diffusion, rotation }\end{array}$ & $\begin{array}{l}\text { Mouvements de } \\
\text { vibration }\end{array}$ & \\
\hline
\end{tabular}
énergies d'excitation des mouvements moléculaires. Ils sont donc un moyen d'investigation très riche pour la matière condensée.

Pour mieux comprendre les mécanismes mis en jeu et leur formulation exacte, il est fortement recommandé de consulter les livres de Lovesey, Squires et Bée, ainsi que les cours précédents des JDN. (cf références en fin de texte).

Dans le cas de la diffusion élastique, il n'y a pas de transfert d'énergie

$$
\hbar \omega=E-E_{0}=0
$$




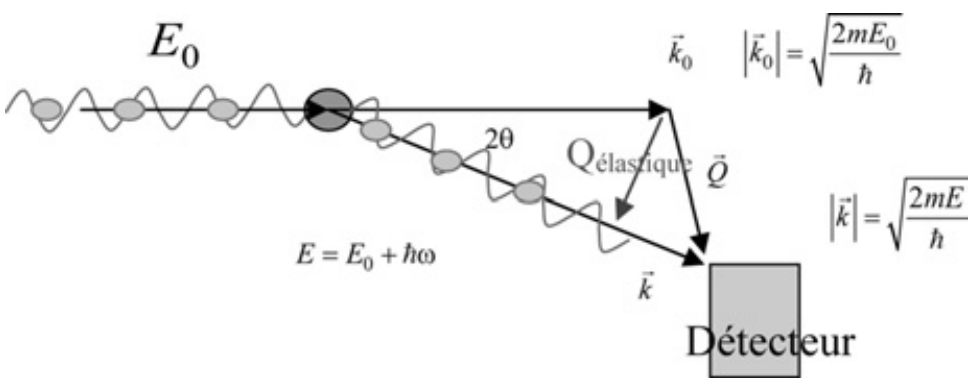

Figure 3. Schéma de principe de diffusion élastique (en bleu) et quasi-élastique (en noir).

et le transfert de moment est

$$
Q_{\text {élastique }}=\frac{4 \pi}{\lambda_{0}} \sin \theta \text { et }|\vec{k}|=\left|\vec{k}_{0}\right|
$$

L'intensité maximale constitue la donnée importante et de nombreuses informations peuvent être extraites de sa dépendance en $Q$; la forme spectrale que l'on mesure alors correspond à la fonction de résolution instrumentale, caractérisée en particulier par sa largeur $\Delta \omega$ (Figure 4(a)) ; cette dernière, généralement mesurée à très basse température $(2-10 \mathrm{~K})$, servira de référence pour normaliser les signaux d'une expérience à l'autre, tel que $\operatorname{Ln} \operatorname{Sel}(Q, \Delta \omega, T) / \mathrm{Sel}(Q, \Delta \omega, T=2 \mathrm{~K})$; cette dernière constitue une des limitations des mesures, le coût d'une meilleure résolution est une diminution de flux et une statistique moindre. Si on se trouve dans des conditions de température suffisante, les vibrations de basse énergie deviennent mesurables en respectant le bilan détaillé (facteur de Bose), l'intensité élastique mesurée à un Q donné diminue, et on observe des processus de diffusion inélastique (Figure 4 (b)) ; la quantité pertinente accessible alors est la densité d'états vibrationnels $g(\omega)$. Enfin si les processus de diffusion sont plus rapides (quelques nano- à picosecondes), alors on considère le cas c de la Figure 4 avec un élargissement quasi élastique qu'il convient de déconvoluer de la fonction instrumentale.

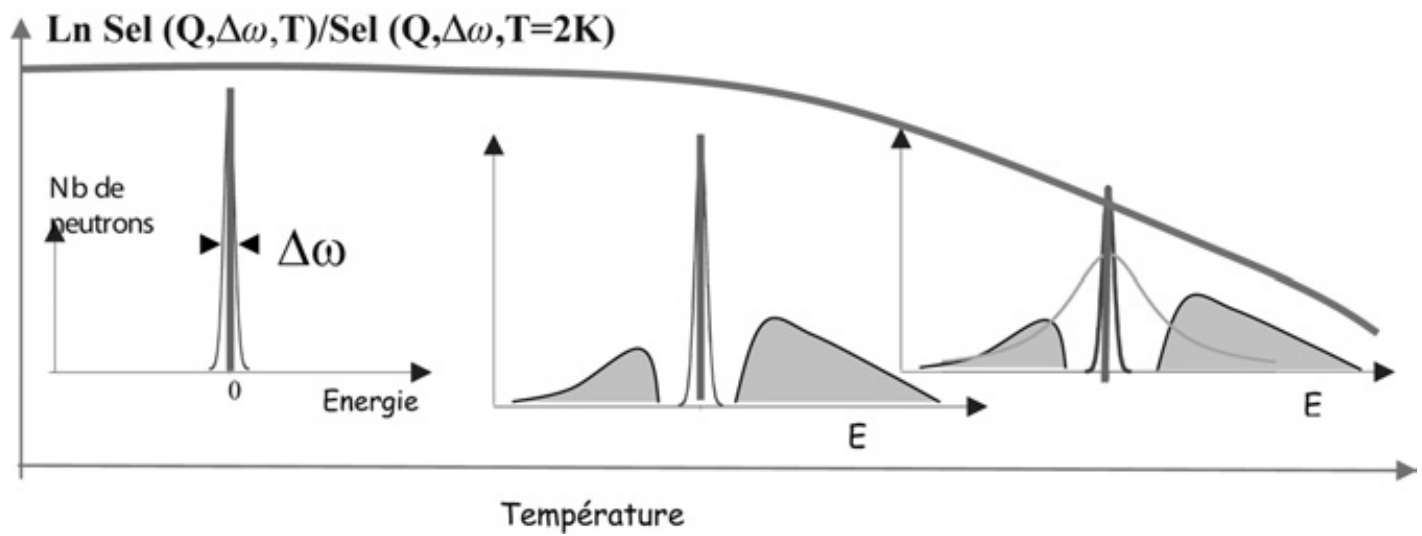

Figure 4. Spectres en énergie obtenus par diffusion de neutrons : (a) case de diffusion élastique et résolution instrumentale ; (b) diffusion élastique et quasiélastique ; (c) mouvements diffusifs et élargissement quasiélastique. 
Les fonctions de diffusion

On se placera ici toujours dans l'approximation classique. La quantité fondamentale permettant de se rapporter à des modèles théoriques est la fonction de van Hove, en partant d'un opérateur densité de particules $\rho(\vec{r}, t)=\sum_{j} \delta\left(\vec{r}-\vec{R}_{j}(t)\right)$ avec $N$ centres diffusants

La fonction de corrélation de paires dépendant du temps soit la probabilité de trouver une particule en $(\vec{r}, t)$ sachant qu'il en avait une en $(0,0)$, pour les phénomènes collectifs $\left(j \neq j^{\prime}\right)$, avec la dimension de l'inverse d'un volume

$$
\begin{aligned}
G(\vec{r}, t) & =\frac{1}{N} \int\left\langle\rho\left(\vec{r}^{\prime}, 0\right) \rho\left(\vec{r}^{\prime}+\vec{r}, t\right)\right\rangle d \vec{r}^{\prime} \\
& =\frac{1}{N} \sum_{j j^{\prime}} \int\left\langle\delta\left[\vec{r}^{\prime}-\vec{R}_{j}(0)\right] \delta\left[\vec{r}^{\prime}+\vec{r}-\vec{R}_{j^{\prime}}(t)\right]\right\rangle d \vec{r}^{\prime}
\end{aligned}
$$

La fonction d'auto-corrélation (ou self) dépendant du temps scrutant les mouvements individuels d'une particule

$$
G_{s}(\vec{r}, t)=\frac{1}{N} \sum_{j} \int\left\langle\delta\left[\vec{r}^{\prime}-\vec{R}_{j}(0)\right] \delta\left[\vec{r}^{\prime}+\vec{r}-\vec{R}_{j}(t)\right]\right\rangle d \vec{r}^{\prime}
$$

Pour obtenir les sections efficaces différentielles partielles, il faut encore effectuer deux transformée de Fourier dans l'espace :

- la fonction de diffusion intermédiaire $F(Q, t)$, aussi écrites $S(Q, t)$ ou $I(Q, t)$, sans dimension

$$
\begin{aligned}
F(\vec{Q}, t) & =\int G(\vec{r}, t) e^{i \vec{Q} \cdot \vec{r}} d \vec{r} \\
& =\frac{1}{N} \sum_{j j^{\prime}}\left\langle e^{-i \vec{Q} \cdot \vec{R}_{j}(0)} e^{i \vec{Q} \cdot \vec{R}_{j^{\prime}}(t)}\right\rangle
\end{aligned}
$$

- la fonction de diffusion intermédiaire self :

$$
\begin{aligned}
F_{S}(\vec{Q}, t) & =\int G_{S}(\vec{r}, t) e^{i \vec{Q} \cdot \vec{r}} d \vec{r} \\
& =\frac{1}{N} \sum_{j}\left\langle e^{-i \vec{Q} \cdot \vec{R}_{j}(0)} e^{i \vec{Q} \cdot \vec{R}_{j}(t)}\right\rangle
\end{aligned}
$$

Il reste encore à faire la même opération dans le temps :

- le facteur de structure dynamique, avec la dimension de l'inverse d'une énergie

$$
\begin{aligned}
S(\vec{Q}, \omega) & =\frac{1}{2 \pi \hbar} \int_{-\infty}^{\infty} F(\vec{Q}, t) e^{-i \omega t} d t \\
& =\frac{1}{2 \pi \hbar N} \int_{-\infty}^{\infty} \sum_{j j^{\prime}}\left\langle e^{-i \vec{Q} \cdot \vec{R}_{j}(0)} e^{i \vec{Q} \cdot \vec{R}_{j^{\prime}}(t)}\right\rangle e^{-i \omega t} d t
\end{aligned}
$$

satisfaisant $\int S(Q, \omega) d \omega=S(Q)$, où $S(Q)$ facteur de structure statique. 
- le facteur de structure dynamique self

$$
\begin{aligned}
S_{\text {inc }}(\vec{Q}, \omega) & =\frac{1}{2 \pi \hbar} \int_{-\infty}^{\infty} F_{S}(\vec{Q}, t) e^{-i \omega t} d t \\
& =\frac{1}{2 \pi \hbar N} \int_{-\infty}^{\infty} \sum_{j}\left\langle e^{-i \vec{Q} \cdot \vec{R}_{j}(0)} e^{i \vec{Q} \cdot \vec{R}_{j}(t)}\right\rangle e^{-i \omega t} d t
\end{aligned}
$$

satisfaisant $\int S_{S}(Q, \omega) d \omega=1$.

Dans une expérience de diffusion quasi élastique, les sections efficaces différentielles mesurées sont constituées d'une partie qui dépend des particularités de l'expérience et des neutrons, et d'une autre partie est caractéristique du système étudié et représente sa fonction de réponse. On obtient donc des informations sur la dynamique collective par la fonction cohérente, et sur la dynamique individuelle par la fonction incohérente de diffusion, dans le cas d'un système isotrope (liquide ou amorphe) :

$$
\left(\frac{d^{2} \sigma}{d \Omega d \omega}\right)_{\mathrm{coh}}=\frac{k}{k_{0}} \frac{\sigma_{c}}{4 \pi} N S(Q, \omega) \text { et }\left(\frac{d^{2} \sigma}{d \Omega d \omega}\right)_{\mathrm{inc}}=\frac{k}{k_{0}} \frac{\sigma_{i}}{4 \pi} N S_{\mathrm{inc}}(Q, \omega)
$$

Par diffusion incohérente, on peut remonter à des grandeurs comme le déplacement carré moyen $\left\langle r^{2}(t)\right\rangle$ ou $\left\langle u^{2}(t)\right\rangle$, le coefficient de diffusion $\mathrm{D}$, séparer des mouvements d'origine vibrationnelle, rotationnelle ou translationnelle selon certaines approximations, ou bien encore dans un solide à la densité d'états vibrationel $g(\omega)$. Si on considère une molécule constituée de $\alpha$ types d'atomes :

$$
\begin{aligned}
\left(\frac{d^{2} \sigma}{d \Omega d \omega}\right)_{\text {inc }}= & \sum_{\alpha} N_{\alpha} K_{\alpha} \frac{1}{2 \pi \hbar} \int_{-\infty}^{+\infty}\left\langle e^{-i \vec{Q} \cdot \vec{R}_{\alpha}(0)} e^{i \vec{Q}^{2} \vec{R}_{\alpha}(t)}\right\rangle e^{-i \omega t} d t \\
\text { avec } K_{\alpha}= & \frac{k}{k_{0}} \frac{\sigma_{i \alpha}}{4 \pi} \text { et } N_{\alpha} \text { le nombre d'atomes } \alpha \text { par unité de volume } \\
& \left\langle e^{-i \vec{Q} \cdot \vec{R}_{\alpha}(0)} \cdot e^{i \vec{Q} \cdot \vec{R}_{\alpha}(t)}\right\rangle=\left\langle e^{i \vec{Q} \cdot\left(\vec{R}_{\alpha}(t)-\vec{R}_{\alpha}(0)\right)}\right\rangle
\end{aligned}
$$

$\vec{R}_{\alpha}(t)=$ position d'un atome quelconque de type $\alpha$, tous équivalents s'ils sont de même type. En considérant le développement en cumulants :

$$
\begin{gathered}
\left\langle e^{A}\right\rangle=e^{\langle A\rangle+1 / 2\left[\left\langle A^{2}\right\rangle-\langle A\rangle^{2}\right]+} \underbrace{1 / 3 !\left[\left\langle A^{3}\right\rangle-3\left\langle A^{2}\right\rangle\langle A\rangle+\langle A\rangle^{3}\right]+1 / 4 !}_{\text {identiquement nul si } A \text { est une variable stochastique gaussienne }} \ldots \\
\text { et }\left\langle\vec{R}_{\alpha}(t)-\vec{R}_{\alpha}(0)\right\rangle=\overrightarrow{0}
\end{gathered}
$$

avec un mouvement isotrope et pas de corrélation des mouvements suivant les axes $x, y, z$ différents, on arrive à

$$
\left\langle e^{-i \vec{Q} \cdot \vec{R}_{\alpha}(0)} \cdot e^{i \vec{Q} \cdot \vec{R}_{\alpha}(t)}\right\rangle=e^{-\frac{Q^{2}}{6}\left\langle\left|\vec{R}_{\alpha}(t)-\vec{R}_{\alpha}(0)\right|^{2}\right\rangle+O\left(Q^{4} \delta R^{4}\right)}
$$

$\delta R$ est l'ordre de grandeur du déplacement ( $\sigma$ pour un liquide) Cette équation (2) est une expression approchée qui ne devient exacte que lorsque $Q \rightarrow 0$ ou $Q \rightarrow \infty$. 
Dans un liquide, les déplacements ne sont pas bornés et on écrit $\vec{R}_{\alpha}(t)-\vec{R}_{\alpha}(0)$ en fonction de la vitesse d'un atome de type, alors

$$
\left.\left.\langle| \vec{R}_{\alpha} t\right)-\left.\vec{R}_{\alpha}(0)\right|^{2}\right\rangle=\frac{6 k_{B} T}{M_{\alpha}} \int_{0}^{t} d t^{\prime}\left(t-t^{\prime}\right) \frac{\left\langle\vec{v}_{\alpha}(0) \cdot \vec{v}_{\alpha}\left(t^{\prime}\right)\right\rangle}{\left\langle\left|\vec{v}_{\alpha}(0)\right|^{2}\right\rangle},\left\langle\left|\vec{v}_{\alpha}\right|^{2}\right\rangle=3 k_{B} T / M_{\alpha}
$$

où $\frac{\left\langle\vec{v}_{\alpha}(0) \cdot \vec{v}_{\alpha}\left(t^{\prime}\right)\right\rangle}{\left\langle\left|\vec{v}_{\alpha}(0)\right|^{2}\right\rangle}$ est la fonction d'autocorrélation des vitesses normalisée.

Dans un solide, les déplacements sont bornés autour de positions figées , alors

$$
\begin{aligned}
\vec{R}_{\alpha}(t)= & \vec{U}_{\alpha}+\vec{u}_{\alpha}(t) \text { avec }\left\langle R_{\alpha}\right\rangle=U_{\alpha} \text {, position figée, } \delta R \approx \sqrt{\left\langle\left|\vec{u}_{\alpha}\right|^{2}\right\rangle} \text { et }\left\langle\vec{u}_{\alpha}(t)\right\rangle=0 \\
& \left\langle\left|\vec{R}_{\alpha}(t)-\vec{R}_{\alpha}(0)\right|^{2}\right\rangle=\left\langle\left|\vec{u}_{\alpha}(t)-\vec{u}_{\alpha}(0)\right|^{2}\right\rangle=2\left[\left\langle\left|\vec{u}_{\alpha}(0)\right|^{2}\right\rangle-\left\langle\vec{u}_{\alpha}(0) \cdot \vec{u}_{\alpha}(t)\right\rangle\right]
\end{aligned}
$$

Cette formule est alors remise dans l'équation (2) puis (1) ; on définit le facteur de Debye Waller pour un atome de type a par

$$
2 W_{D W}^{(\alpha)}(Q)=\frac{Q^{2}}{3}\left\langle\left|\vec{u}_{\alpha}\right|^{2}\right\rangle+O\left(Q^{4}\left\langle\left|\vec{u}_{\alpha}\right|^{2}\right\rangle^{2}\right)
$$

On peut développer l'exponentielle sous l'intégrale car $Q^{2} \delta R^{2}<<1$, ce qui fait apparaître un terme élastique, la contribution à un phonon et les contributions à plusieurs phonons

$$
e^{-\frac{Q^{2}}{3}\left\langle\vec{u}_{\alpha}(t) \cdot \vec{u}_{\alpha}(0)\right\rangle+O\left(Q^{4} \delta R^{4}\right)}=1+\frac{Q^{2}}{3}\left\langle\vec{u}_{\alpha}(t) \cdot \vec{u}_{\alpha}(0)\right\rangle+O\left(Q^{4} \delta R^{4}\right)
$$

Et on arrive à

$$
\left(\frac{d^{2} \sigma}{d \Omega d \omega}\right)_{\text {inc }}=\sum_{\alpha} N_{\alpha} K_{\alpha} e^{-W_{D W}^{(\alpha)}(Q)}\left[\delta(\omega)+Q^{2} \frac{k_{B} T}{2 M_{\alpha}} \frac{g_{\alpha}(\omega)}{\omega^{2}}\right]
$$

où $g_{\alpha}(\omega)$ est la densité d'états vibrationnel relative aux atomes de types $\alpha$.

\subsection{Les spectromètres}

Pour découvrir les divers types de spectromètres (trois axes, temps de vol, rétrodiffusion et écho de spin) et leurs caractéristiques, il est conseillé de rendre directement sur les sites web des centres de neutrons où les explications sont claires et détaillées. Le domaine d'énergie couvert est de l'ordre du $\mu \mathrm{eV}$ à $100 \mathrm{meV}$, soit de quelques picosecondes à 100 nanosecondes, dans une gamme de $\mathrm{Q}$ de 6 à $0.01 \AA^{-1}$.

Spectrométre à temps de vol (TOF)

Cet appareil permet de détecter les neutrons diffusés, selon leur angle et leur énergie, et ainsi d'obtenir le facteur de structure dynamique $S(2 \theta, \omega)$. Des neutrons de la longueur d'onde désirée sont sélectionnés. Cette sélection peut se faire tant par l'utilisation de monocristaux, que par des "choppers" successifs, disques ne laissant passer par une fente que les neutrons de vitesse donnée.

\section{Spectromètres à haute résolution (à rétrodiffusion et à écho de spin, BS et NSE)}

Les spectromètres à rétrodiffusion et à écho de spins (NSE et RNSE à écho de spin par résonance ou en champ nul) sont les instruments de plus haute résolution en diffusion neutronique ; ils permettent d'avoir accès aux processus les plus lents. Comme pour le TOF, les spectromètres à rétrodiffusion 
(BackScattering) couvrent une large gamme de vecteurs d'onde avec une excellent résolution en Q, et en énergie $(\sim 1 \mu \mathrm{eV})$, mais sur une gamme d'énergie plus petite $( \pm 30 \mu \mathrm{eV})$; la diffusion incohérente est plus favorable. Les spectromètres à écho de spins permettent de mesurer directement la fonction de diffusion intermédiaire, donc dans le temps ; cette méthode utilise les propriétés magnétiques associées au spin du neutron. Ce sont des instruments pour lesquels la mesure du changement de vitesse du neutron avant et après le processus de diffusion se fait en étudiant le nombre de rotations de Larmor que font les spins des neutrons en passant dans une zone de champ magnétique. Le domaine dynamique scruté est large (de l'ordre de 3 à 4 décades en temps), mais le faisceau est faiblement monochromatique $(\Delta \lambda / \lambda \sim 15 \% \sim \Delta Q / Q)$ et les résultats sont moins bien résolus en Q. La diffusion cohérente est la mieux adaptée dans ce cas.

Si l'on veut étendre le domaine en fréquence ou en temps (par exemple incohérent TOF + BS, cohérent TOF + NSE), il est conseillé de combiner les spectromètres à TOF avec la rétrodiffusion ou l'écho de spin. Cependant, on rencontre un autre type de difficultés pour le raccordement inhérentes aux deux techniques expérimentales : la quantité de neutrons diffusés provient de la combinaison des contributions cohérente et incohérente avec des pondérations différentes selon les méthodes. Si on souhaite extraire exactement l'une ou l'autre, il est nécessaire de combiner les deux types de mesures BS ou NSE

Ainsi pour TOF, BS

$$
S_{\exp }(Q, \omega)=\sigma_{\text {coh }} S_{\text {coh }}(Q, \omega)+\sigma_{\text {inc }} S_{\text {inc }}(Q, \omega)
$$

Et pour l'écho de spin (et d'une façon générale dès que l'on utilise une technique de polarisation de neutrons)

$$
S_{\mathrm{NSE}}(Q, t)=\frac{\sigma_{\mathrm{coh}} S_{\mathrm{coh}}(Q, t)-\frac{1}{3} \sigma_{\mathrm{incoh}} S_{\mathrm{incoh}}(Q, t)}{\sigma_{\mathrm{coh}}-\frac{1}{3} \sigma_{\text {incoh }}}
$$

\subsection{Corrections et méthodes d'analyse}

Les corrections standard suivantes sont généralement proposées à l'issue des expériences sur le site, elles contiennent les caractéristiques de l'instrument nécessaires. Elles consistent par exemple en -la détermination de la position du pic élastique : cette première étape est nécessaire pour la conversion du spectre en énergie, cette position sera définie comme l'origine (zéro des énergies), -la correction de l'efficacité relative des détecteurs par du vanadium, -la soustraction de la cellule vide ou-la correction d'absorption selon la géométrie de la cellule. Cela permet d'avoir des fichiers des sections efficaces différentielles fonction de 1 angle de diffusion et de l'énergie ; mais il faudrait tenir compte en plus des effets de la diffusion multiple pour se permettre de les négliger ce qui est fait dans la plupart des cas, des contributions à plusieurs phonons dans le cas de solides ou de verre.

Dans le cas des expériences en temps de vol, le facteur de structure dynamique obtenu est fonction

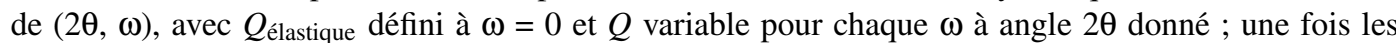
soustractions nécessaires faites, pour pouvoir évaluer un modèle et faut convertir les données en $(Q, \omega)$, selon la formule d'interpolation suivante, où $E_{i}$ est l'énergie incidente du neutron, ce qui restreint le domaine d'analyse :

$$
Q(\hbar \omega)=\left(\frac{2 m_{n}}{\hbar^{2}}\left[2 E_{i}-\hbar \omega-2 \cos (2 \theta) \sqrt{E_{i}^{2}-\hbar \omega E_{i}}\right]\right)^{1 / 2}
$$

Il faut mesurer la fonction de résolution instrumentale dans les conditions de l'échantillon, tenir compte du rapport signal/bruit et de la forme de la résolution du spectromètre $R(\omega)$ pour extraire le facteur de structure dynamique de l'échantillon.

$S_{\exp }(Q, \omega)=S_{\text {theorique }}(Q, \omega) \otimes R(\omega)$ si on a un modèle pour réaliser la convolution ou bien après transformation de Fourier :

$$
\text { T.F. }\left[S_{\exp }(Q, \omega)\right]=S_{\text {théorique }}(Q, t) \bullet R(t)
$$


Pour les expériences en TOF, il faut encore diviser par l'intégrale de $S(Q, \omega)$ en fréquence pour obtenir in fine $F(Q, t)$ normée entre 1 et 0 ; c'est cette dernière quantité qui est obtenue directement dans le cas des expériences par NSE.

Mais quelle soit la mesure faite en fréquence ou en temps, il faut alors considérer la contribution de la matrice vide. Ceci représente une difficulté supplémentaire par rapport au traitement standard proposé ci-dessus. La matrice est prise en compte dans la mesure globale et elle participe fortement au signal élastique, lorsque celle-ci est solide. Si la matrice d'une part et le fluide confiné d'autre part se rapportent à des processus statistiquement indépendants, alors une simple soustraction suffit ; toutefois il est conseillé de le faire directement en $2 \theta$ pour le TOF, ou directement sur l'écho et son amplitude par NSE, (le quantités sont additives mais pas leur combinaison).

Par ailleurs, il y a des matériaux même vides et propres, pour lesquels des contributions quasiélastiques peuvent apparaître dues à des groupements de surface (de l'eau difficile à extraire), ou à la diffusion d'ions, de défauts. Il faut donc les prendre en compte dans l'analyse de la matrice vide, mais elles peuvent aussi s'exprimer différemment après remplissage et ces mouvements peuvent se coupler à la dynamique de surface des molécules (cf Figure 1). Plusieurs stratégies sont possibles : on peut mesurer le matériau vide dans les mêmes conditions qu'avec l'échantillon, on peut mesurer l'ensemble (fluide + matériau) à haute température où on estime que la contribution du fluide n'est plus dans la fenêtre spectrale, on peut la prendre en compte globalement avec un paramètre de fit particulier.

Enfin, la présence d'un mur rigide, ralentissant ou adsorbant des molécules à la surface, favorise souvent la constitution d'une couche apparemment figée dans la fenêtre spectrale et participe au phénomène physique qui nous intéresse et il est difficile de ne pas la confondre avec la matrice. Alors des mesures complémentaires par RMN, avec des sondes temporelles plus lentes, sont à envisager par exemple.

\section{DIFFUSION QUASI-ÉLASTIQUE DE NEUTRONS EN MILIEU CONFINÉ : CAS D 5 À $20 \sigma$ (TAILLE DE LA MOLÉCULE)}

\subsection{Conséquences du confinement nanoscopique sur la dynamique de liquides}

La présence d'un mur rigide plus ou moins attractif (selon les groupements de surface que l'on peut greffer post-synthèse) entraîne un ralentissement des molécules à la surface et impose une distribution

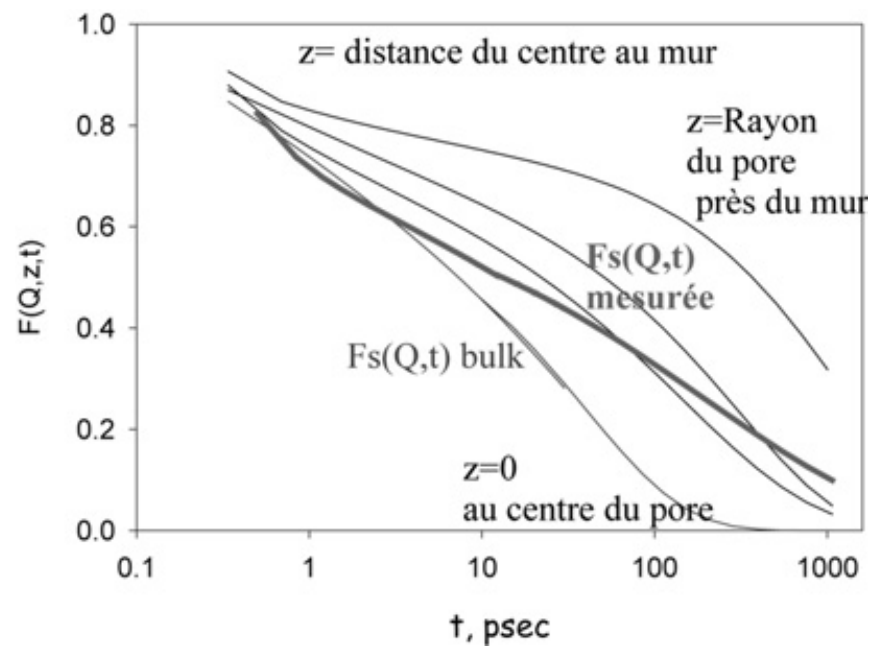

Figure 5. Représentation schématique des fonctions de diffusion intermédiaires à un vecteur d'onde et une température donnés, pour des molécules situées à des distances différentes au mur ; la $F s(Q, t)$ du liquide en volume est comparée. 
des temps de relaxations à l'intérieur du pore, donc une dynamique de facto spatialement hétérogène. La conséquence en est un plus grand étalement des fonctions de relaxation qui sont moyennées dans tout le pore et un temps de relaxation moyen plus lent que celui en volume dans les mêmes conditions. Les fonctions peuvent être ajustées soit par une exponentielle étirée (fonction de Kohlrausch) soit en introduisant une distribution de temps particulière, dont on ne peut pas extraire d'informations spatiales à ce niveau de traitement.

$F s(Q, t)$ est une valeur moyenne résultante de la somme des différentes $F s(Q, r, t)$ dépendantes de la distance du centre du pore au mur, les contributions de $F s(Q, r, t)$ sont pondérée par le nombre de molécules impliquées, nombre que l'on peut connaître ( par simulation numériques) à partir des profils de densité radial, distribution des populations à l'intérieur du pore est justement une quantité à laquelle il serait intéressante de remonter expérimentalement. A des températures où le liquide a un temps de relaxation de l'ordre de quelque picoseconde, les molécules situées au centre se subissent pas la présence du mur et auraient si on pouvait les mesurées individuellement, des temps de relaxation proche de celles du système en volume.

Ceci est illustré par simulation numérique de la dynamique moléculaire du toluène liquide piégé dans des cylindres dont les murs sont constitués de molécules de toluène figés afin de réduire les interactions de surface. On constate à $200 \mathrm{~K}$ une différente de temps de relaxation de plus de 2 décades entre les molécules au centre du pore et celles près du mur.

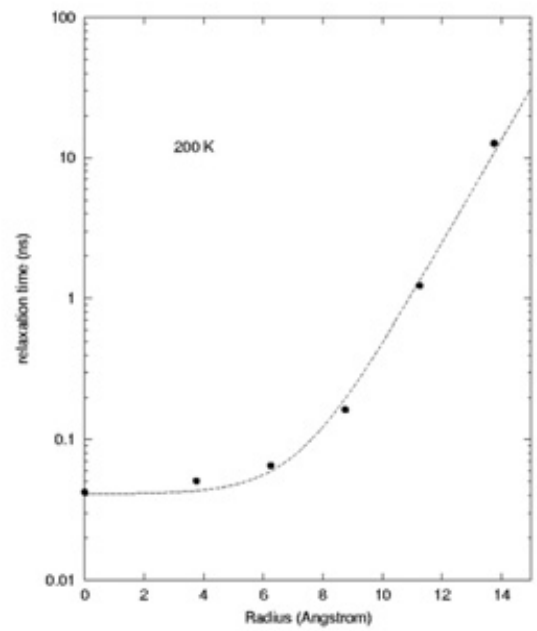

Figure 6. Temps de relaxation du toluène liquide à l'intérieur de cylindres de toluène par simulations numériques : dynamique spatialement hétérogène (V. Teboul). Les temps sont extraits ici en prenant $F s(Q, t)=1 / e$ à $\tau_{\alpha}$.

On observe ce ralentissement dans le cas où le confinement correspond à quelques diamètres moléculaires (de 3 à 10 diamètres moléculaires $\sigma$ ), ce dernier est à prendre en compte comme un facteur d'échelle lorsque l'on compare plusieurs liquides confinés, si tant est que les interactions avec le mur soient comparables.

S'il y a une grande séparation des échelles de temps entre les molécules du centre et celles du bord, alors on peut observer les deux populations sous la forme suivante : la fonction intermédiaire contient un fond élastique de molécules qui ne contribuent pas au phénomènes de diffusion et le processus de relaxation ralentie :

$$
F s(q, t)=(1-f q) \exp \left(-{ }^{(t / \tau q)^{\beta q}}\right)+f q * A
$$

Ce fond élastique varie avec la température, signalant le nombre de plus en plus grand de molécules ralenties à la surface et avec le degré de confinement. Les figures suivantes illustrent cette situation pour 
la diffusion incohérente et pour la diffusion cohérente du toluène dans des matériau de type MCM-41 et SBA-15, sans traitement de surface.
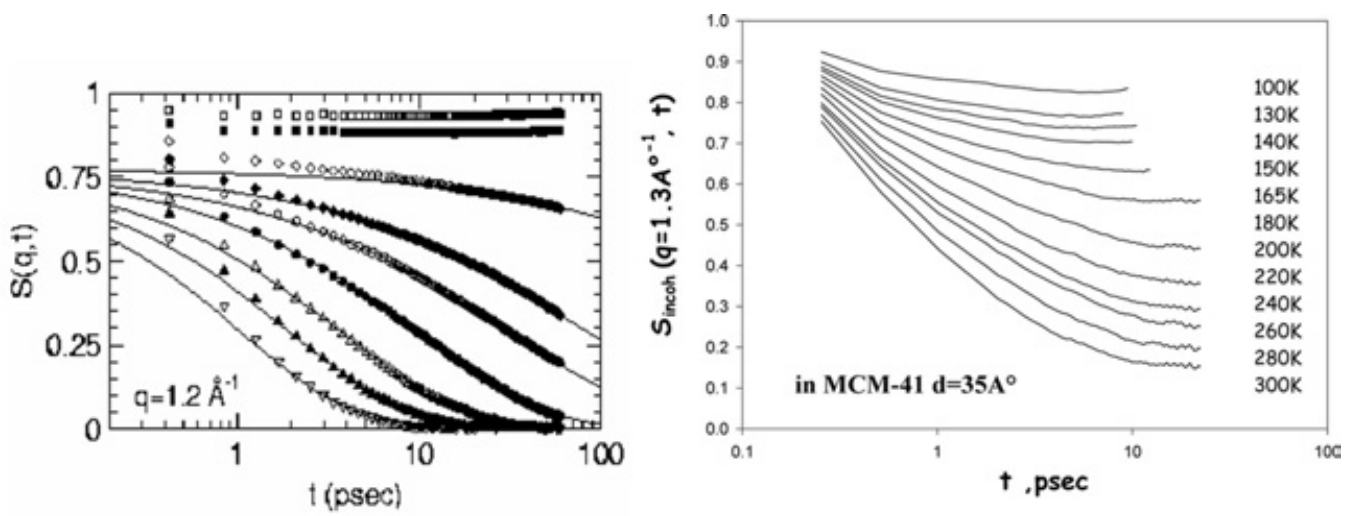

Figure 7. Fonctions de diffusion intermédiaires obtenues après transformation de Fourier de $S(Q, w)$ obtenus par temps de vol (IN6 à l'ILL) à $q=1.2 \AA^{-1}$; (a) toluène bulk à différentes tempèratures (de haut en bas, $79 \mathrm{~K}, 112 \mathrm{~K}$, $143 \mathrm{~K}, 160 \mathrm{~K}, 168 \mathrm{~K}, 189 \mathrm{~K}, 209$ K, 239 K, 284 K) (b) toluène confiné dans une MCM-41 dans la même gamme de température ; on n'observe pas de retour à 0 de la fonction de corrélation et un fond élastique croissant continûment avec $T$.

Si on s'intéresse maintenant à la dynamique collective du toluène confiné nano-sec) avec la fonction intermédiaire cohérente $S(Q, t)$ (NSE, $\left.Q=1.3 \AA^{-1} \mathrm{CoM}\right), 180 \mathrm{~K}$ ), on constate que lorsque le diamètre du pore diminue de 12 de diamètres moléculaires à quelques $\sigma$, la dynamique est ralentie par plus d'un ordre de grandeur (Figure 8), ce qui correspond à une diminution de température en volume de 30 degrés.
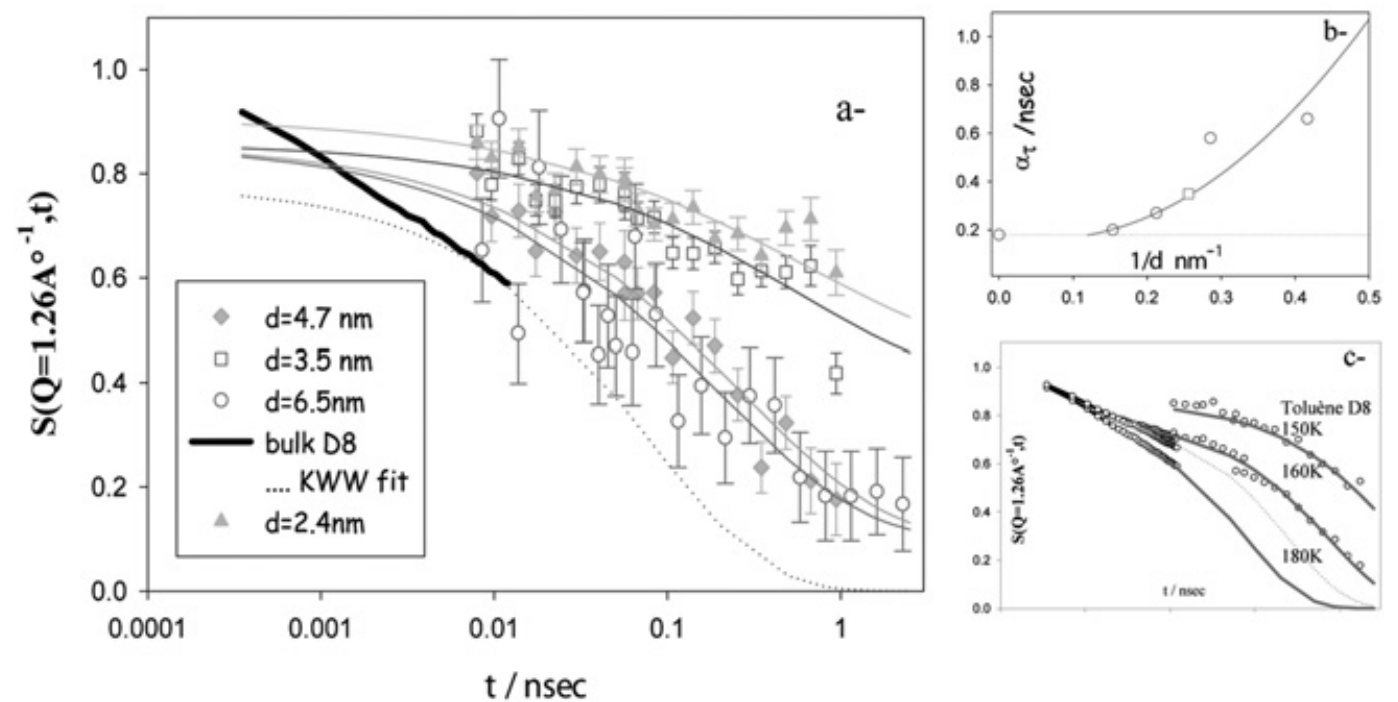

Figure 8. Dynamique collective du toluène D8 : (a) confiné dans des matrices de type MCM-41 et SBA-15 avec des diamètres de pores de 65 à $24 \AA, F(Q, t)$ par NSE à $180 \mathrm{~K}$; (b) temps de relaxation correspondant à $F(Q, t)$ confiné à $180 \mathrm{~K}$ dans diverses tailles de pores : (c) comparaison au toluène $D 8$ en volume à différentes températures $180 \mathrm{~K}, 160 \mathrm{~K}, 150 \mathrm{~K}$ à $Q=1.3 \AA^{-1}$ qui correspond au maximum du facteur de structure du center de masse(CoM) de la molécule. 
Enfin une des grandeurs communément traitées en milieu confinés correspond à un déplacement carré moyen effectif $\left\langle u^{2}\right\rangle$ obtenu à partir de l'intensité élastique à $\omega=0$ mesurée à différents $Q$ par rétrodiffusion, ou bien par temps de vol. L'intensité élastique est très sensible à l'apparition d'une contribution quasi-élastique ; elle permet d'obtenir une information qualitative de ce qui se passe dans le système, et signalera l'apparition de mouvements plus rapides que la résolution instrumentale, typiquement des mouvements plus rapides quelques nanosecondes par BS et quelques 30picoseconde par temps de vol.

$$
\begin{aligned}
S_{\mathrm{el}}(q, \Delta \omega, T) / S_{\mathrm{el}}(q, \Delta \omega, T=0) & =\exp \left[-2 W(q, \Delta \omega, T]=\exp \left[\left\langle-u^{2}(T)\right\rangle q^{2} / 3\right]\right. \\
\left\langle u^{2}\right\rangle_{e f f} & \propto k_{B} T \int_{0}^{\omega_{m}} \frac{1}{\omega^{2}} g(\omega) d \omega \propto T
\end{aligned}
$$

Dans un domaine de température où le système est solide, alors ce $\left\langle\mathrm{u}^{2}\right\rangle$ varie linéairement avec la température puis augmente plus rapidement au passage de la température de transition vitreuse $T_{g}$. Celleci varie avec la taille du pore : elle est d'autant plus élevée que le diamètre diminue. Notons que dans certains cas après des traitements de surface, $T_{g}$ apparemment est inférieure à sa valeur en volume. L'amplitude des mouvements est aussi considérablement réduite dans le cas des plus fortes restrictions géométriques.

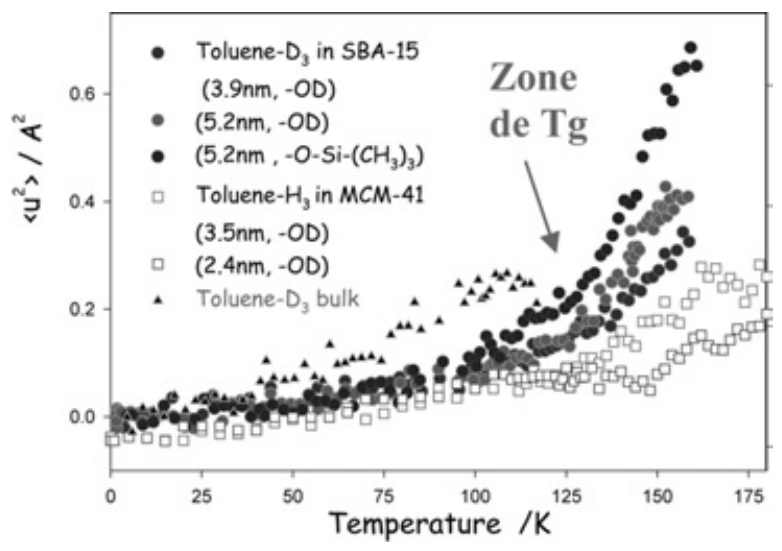

Figure 9. Variations déplacement carré moyan $\left\langle\mathrm{u}^{2}\right\rangle$ pour du toluéne confiné dans des matrices silicatées mesurés par rétro-diffusion (sur IN16 à ILL) en fonction de la température.

Pour interpréter plus finement ces résultats, nous devrons nous situer selon deux cas limites comme cela a été introduit ci-dessus et utiliser des mesures complémentaires d'adsorption, de thermodynamique, de spectroscopie diélectrique ou RMN, simulations numériques.

\subsection{Approches théoriques et modèles compatibles}

Si nous nous plaçons dans le cas où la dynamique est profondément modifiée par des effets de taille finie et la présence d'une surface, il existe deux approches dans la littérature.

- La présence d'un mur rigide induit un ralentissement graduel des molécules relatif à leur distance au mur. De ce ralentissement dans l'espace on peut extraire une longueur de nature coopérative $\xi_{\text {coop }}$. C'est une approche suivie pour les résultats de simulations numériques où l'intérieur du pore peut être découpé en tranches. 
La fonction intermédiaire incohérente est considérée avec cette variable additionnelle, $\rho$ représentant la distance au mur par rapport à l'axe z du pore :

$$
F_{S}(q, \rho, t)=\left\langle\exp \left[i q, \cdot\left(r_{j}(t)-r_{j}(0)\right)\right] \delta\left(r_{j}(0)-\rho\right)\right\rangle
$$

Le temps de relaxation associé à ces fonctions suit une loi de type $\left(\Delta_{q}\right.$ et $\rho_{P}$ paramètres de fit $)$ :

$$
\tau(q, \rho, T)=C(q) f(T) \exp \left[\Delta_{q} /\left(\rho_{P}-\rho\right)\right]
$$

Cette loi serait valide surtout près du mur

Le point de croisement entre cette loi et la valeur plus près du centre égale à la celle du bulk définit une longueur de coopérativité spécifique au système confiné.

Un « ansatz » de cette formule, sans passer par le calcul du temps de relaxation, est de considérer l'écart entre la fonction $F s(Q, \rho, T)$ et celle du liquide en volume.

$$
\left.F s(Q, r, t)=F s^{\text {bulk }}(Q, t)\right)+a(t) \exp \left[-(r / \xi(t))^{\beta(t)}\right]
$$

Dans ce cas, c'est toute la dépendance en $r$ de la fonction qui est prise en compte par une exponentielle étirée.

- Une deuxième approche est traduite dans la théorie de couplage de modes en milieu confiné (Krakoviack PRL 2005). La théorie de couplage de modes (Götze 1984) cherche à rendre compte de la dynamique d'un système physique à partir de variables lentes bien choisies et de leur produit. Les variables lentes sont composantes de Fourier des fluctuations de densités associées aux grandeurs conservées (nombre de particules, quantité de mouvement, énergie). Cette théorie est particulièrement bien adaptée à des systèmes simples monoatomiques, à l'échelle des distances inter-atomiques et hors de la limite hydrodynamique. Le ralentissement visqueux et la transition vitreuse sont traités par analogie aux les phénomènes critiques avec une mise en équation d'un mécanisme de rétroaction comme source de ralentissement, dans un formalisme de fonction mémoire. La théorie prédit certaines lois de puissances pour les processus de relaxation et une température de transition $T_{c}$, située très au-dessus de $T_{g}$, signalant le passage d'un état d'équilibre ergodique du système, le liquide surfondu, à un état non-ergodique et hors d'équilibre, le verre.

Une version de la théorie de couplage de modes en volume a été récemment adaptée pour la dynamique des liquides en milieux confinés sur la base d'un mélange binaire «quenched-annealed »; elle rend compte de l'évolution du fond élastique observé expérimentalement. En effet, les fluctuations de densité ne retournent jamais à 0 même dans la phase ergodique. Dans cette théorie, il suffit de bien connaître la structure pour pouvoir prédire la dynamique, et regarder la partie connectée des fonctions de structure et non les corrélations totales. Un nouveau scénario de transitions est prédit incluant le passage d'une transition discontinue de type $\mathrm{B}$, tel qu'on l'observe en volume, à une transition continue de type $\mathrm{A}$, où la relaxation est en une seule étape dans la phase liquide.

\subsection{Autres modèles compatibles}

Dans la situation où la dynamique n'est pas intrinsèquement modifiée, mais c'est l'étendue des déplacements qui se retrouve est limitée par les restrictions géométriques ; ceci conduit à tronquer l'espace et introduire seulement des effets de volume exclu. Il existe plusieurs modèles dans la littérature pouvant servir à l'analyse des processus dynamiques mis en jeu dans un spectre de diffusion quasi-élastique. Citons parmi eux :

-La diffusion par sauts, qui s' applique aux grands $Q$ (cours de M.Bée) On définit $\tau_{0}$ un temps de résidence dans un site $\mathrm{D}$ et coefficient de diffusion est obtenu par :

$$
S_{\text {inc }}(Q, \omega)=\frac{1}{\pi} \frac{f(Q)}{(f(Q))^{2}+\omega^{2}} \operatorname{avec} f(Q)=\frac{D Q^{2}}{D Q^{2} \tau_{0}+1}
$$


-L'ajustement d'un EISF (Elastic Incoherent Structure Factor) :

Dans cette méthod, on considère $F s$ à $t \xi$ et on décompose le spectre en plusieurs composantes lorentziennes pondérées par un facteur EISF dépendant de $Q$ L'intensité d'un spectre de diffusion incohérente décrit l'environnement statique de la particule piégée.

Dans cette méthod, le modèle souvent utilisé est celui de Volino et Dianoux. Considérant la diffusion libre d'une particule dans une sphère de rayon a : la particule est en mouvement dans un potentiel sphérique $V(r)$, avec $V(r)=0$ pour $0<r<a$ et $V(r)=\infty$ pour $r>a$

$$
S_{\text {inc }}(Q, \omega)=A_{0}^{0}(Q) \delta(\omega)+\Sigma \text { lorentziennes }
$$

Ce modèle de diffusion à l'intérieur d'un pore, généralisable à des géométries diverses, a été à nouveau proposé en 2006 (Perrin J.C. et al 2006) avec une formulation simple.

-Une diffusion unidimensionnelle (cf cours H. Jobic)

$$
S_{1 D}(Q, \omega)=\frac{1}{2 \pi} \int_{0}^{\pi} \frac{D Q^{2} \cos ^{2} \theta \sin \theta}{\left(D Q^{2} \cos ^{2} \theta\right)^{2}+\omega^{2}} d \theta
$$

Quand le diamètre du pore est de l'ordre de grandeur de la molécule, on considère le cas de diffusion à la file indienne

Toutes ces méthodes sont trés largement tilisées pour analyser les donées de dynamique obtenues par les expériences de diffusion quasi-élastique de neutrons.

\section{Références}

\section{Simulations numériques :}

Scheidler, P., Kob et W., Binder, K., J. Phys. Chem. B 108, 6673 (2004).

Scheidler, P., Kob, W., Binder, K., Europhysics Letters 2002, 59, 701-707.

Scheidler, P., Kob, W., Binder, K., European Physical Journal E 2003, 12, 5-9.

Coasne, B., Pellenq, R. J. M., Journal of Chemical Physics 2004, 120, 2913-2922.

Gallo, P., Pellarin et R., Rovere, M., Europhys. Lett. 57, 212 (2002)

Teboul, V., Alba-Simionesco, C., J. Phys. : Condens. Matter 14, 5699-5709, (2002) ; Chem. Phys. 317, 245 (2005)

\section{Propriétés structurales et dynamiques en milieu confiné :}

McKenna, G. B., Journal de Physique IV 2000, 10, 343.

Forrest, J. A., Dalnoki-Veress, K., Advances in Colloid and Interface Science 2001, 94, 167-4196.

Forrest, J. A., European Physical Journal E 2002, 8, 261-266.

Wang, L. M., He, F., Richert, R., Phys. Rev. Lett. 2004, 92, 95701.

Frick, B., Alba-Simionesco, C., Dosseh, G., Le Quellec, C., Moreno, A. J., Colmenero, J., Schönhals, A., Zorn, R., Chrissopoulou, K., Anastasiadis, S. H., Dalnoki-Veress. K.,(2005) Inelastic neutron scattering for investigating the dynamics of confined glass forming liquids, Journal of Non-Crystalline Solids 351 (2005) 2657-2667

Guégan, R., Morineau, D., Alba-Simionesco C., (2005) Interfacial structure of an H-bonding liquid confined into silica nanopore with surface silanols, Chem.Phys. 317.

Dosseh, G., Brodie-Linder, N., Frick, B., Le Quellec, C., Morineau, D., Alba-Simionesco C., (2005) Dynamical properties of toluene and ortho-terphenyl confined in MCM-41 and SBA-15 mesoporous materials. Ann. Chim. Sci. Mat. 2005, 30 (4), pp 365-373.

Zorn, R., Frick, B., Hartmann, L., Kremer, F., Richter, D., Physica B 2004, 350.

Schönhals, A., Goering, H., Schick, C., Frick, B., Zorn, R., Eur. Phys. J. E 12 (2003) 173.

Richert, R., Min, Y., J. Phys. Chem. B 107 (2003) 895. 
Morineau, D., Xia, Y., Alba-Simionesco, C., J. Chem. Phys. 117 (2002) 8966.

Dosseh, G., Le Quellec, C., Brodie-Linder, N., Alba-Simionesco, C., Haeussler, W., Levitz, P., J. Non Cryst. Solids (2006).

Yongde Xia, Gilberte Dosseh, Denis Morineau, and Christiane Alba-Simionesco, J. Phys. Chem. B 2006. Jackson et, C. L., McKenna. G. B., Chem. Mater. 8, 2128 (1996).

Alba-Simionesco, C., Dosseh, G., Dumont, E., Frick, B., Geil, B., Morineau, D., Teboul, V., Xia. Y., Eur. Phys. J. E 12 19-28 (2003).

\section{Revues sur les effets du confinement}

Alcoutlabi, M. and McKenna, G. B., 2005 J. Phys. : Condens. Matter 17 R461.

Alba-Simionesco, C., Coasne, B., Dosseh, G., Dudziak, G., Gubbins, K. E., Radhakrishnan, R. and Sliwinska-Bartkowiak, M., Topical review : Effects of confinement on freezing and melting J. Phys. : Condens. Matter 18 (2006) R15-R68.

In Journal de Physique IV ; Frick, B., Büttner, H., Zorn, R., eds., EDP SCIENCES : Les Ulis, France, 2000 ; Vol. 10.

In European Physical Journal E ; Frick, B., Koza, M., Zorn, R., eds., 2003 ; Vol. 12, pp 3-4.

In Proceedings of the MRS ; Fourkas, J. T., Levitz, P., Urbakh, M., Wahl, K. J., eds., MRS : Boston, 2003 ; Vol. 790, p 336.

In European Physical Journal E ; Frick, B., Koza, M., Zorn, R., eds., 2006 ;

\section{Diffusion de neutrons}

Lovesey, S. W. Theory of Neutron Scattering from Condensed Matter ; Clarendon Press : Oxford, 1984. http://www.ncnr.nist.gov/resources/n-lengths/.

Mezei, F., The Principles of Neutron Spin Echo; Springer Verlag : Grenoble, 1979 ; Vol. 112.

Frick, B., Farago, B., In Scattering ; Sabattier, P., Ed. ; Academic Press, 2002 ; Vol. 2, chapter 2.8.4, pp 1209-1241.

Bée, M., Quasielastic Neutron scattering ; Adam Hilger : Bristol, 1988.

Angell, C. A., Ngai, K. L., G. B., Zorn, R., in Neutrons, X-ray and Light : Scattering Methods Applied to Soft Condensed Matter, edited by Lindner, P. and Zemb, T., (North-Holland, Amsterdam, 2002).

ILL, (http://www.ill.fr/tof/lis_prog_tof.html).

Van Hove L., Phys. Rev. 95, 249 (1954)

de Gennes P.-G., Physica 25, 825 (1959).

Sköld, K., Rowe, J. M., Ostrowski, G., Randoph, P.D., Phys. Rev. A 6, 1107 (1972).

Schärpf, O., Capellmann, H., Phys. Satus Solidi A 135, 359 (1993).

Mezei, F., in "Liquids, Freezing, and the Glass Transition", Hansen, J. P., Levesque, D. and -Zinn-Justin J., eds. (North Holland, Amsterdam, 1991), pg. 629.

Tölle, A., Rep. Prog. Phys. 64, 1473-1532 (2001).

Cours des JDN : Bee, M., Dianoux dans J., J. Phys. IV France 111 (2003), Neutrons et systèmes désordonnés ; Zanotti dans, J. M., J. Phys. IV France 111 (2005), Neutrons et Biologie.

Neutron and Synchrotron radiation for condensed matter studies Volume I, Theory,

Instruments and Methods Cours Hercules, 2001.

Volino, F., Dianoux, J., Mol. Phys. 41, 271-279 (1980)

Perrin, J.C, Lyonnard, S., Volino F., Guillermo A., Euro. Phys. J. 2006 